\title{
SOBOLEV COHOMOLOGY FOR LOCALLY POLYCYLINDRICAL DOMAINS
}

\author{
PATRICK W. DARKO
}

Received 13 April 2001

The Cauchy-Riemann equation is solved with $L^{p}$-Sobolev space estimates and the result is used to obtain vanishing theorems on locally polycylindrical domains.

2000 Mathematics Subject Classification: 32W05, 32W10, 35N15.

1. Introduction. In [1], Andreotti and Stoll established vanishing theorems for uniform cohomology on polycylinders and, in [6], Nagel generalized their result to $L^{p_{-}}$ cohomology $1 \leq p \leq \infty, C^{k}$-cohomology, and Hölder cohomology. The obvious classical spaces that were left out are the Sobolev spaces. In this paper, we prove vanishing theorems for Sobolev cohomology on polycylinders and, at the same time, extend our results to a particular generalization of polycylinders called locally polycylindrical domains. A polycylinder $\Omega$ in $\mathbb{C}^{n}$ is a domain of the form $\Omega=D_{1} \times D_{2} \times \cdots \times D_{n}$, where each $D_{j}$ is a nonempty, bounded open subset of $\mathbb{C}$. And a bounded open set $\Omega$ is a locally polycylindrical domain, if for each point $x$ in the boundary $\partial \Omega$ of $\Omega$, there is an open neighborhood $U_{x}$ of $x$ in $\mathbb{C}^{n}$ such that $\Omega \cap U_{x}$ is a polycylinder. A locally polycylindrical domain is obviously Stein.

We use the method of establishing estimates for the $\bar{\partial}$-operator, developed in [3]. This method has the advantage of giving results on polycylinders more general than the polycylinders considered in [6]. We then use Leray's isomorphism theorem and Dolbeault isomorphism theorem with bounds to obtain a solution of the $\bar{\partial}$-equation on locally polycylindrical domains, from which we obtain the required vanishing theorems.

A polycylinder is called admissible if it has each factor in $\mathbb{C}$ with a boundary with plane measure zero. We assume throughout this paper that all polycylinders mentioned are admissible.

\section{Preliminaries}

2.1. In this section, we define some of the terms that we are going to use and state our main results. Since a polycylinder is a locally polycylindrical domain, we state the vanishing theorem for locally polycylindrical domains only.

Let $U$ be an open set in $\mathbb{C}^{n}$ and let $L^{p, s}(U), 1 \leq p \leq \infty$, and $s$, a nonnegative integer, denote the space of functions in $U$ whose distributional derivatives of order $\leq s$ are in $L^{p}(U)$.

For $f \in C^{\infty}(U)$, define

$$
\|f\|_{L^{p, s}(U)}^{(0)}=\|f\|_{L^{p, s}(U)}^{(0,0)}=\|f\|_{L^{p, s}(U)},
$$


$\|f\|_{L^{p, s}(U)}$ as the $p$-s Sobolev norm of $f$ on $U$,

$$
\begin{aligned}
& \|f\|_{L^{p, s}(U)}^{(0, r)}=\max _{i_{1}<\cdots<i_{r}}\left\|\frac{\partial^{r} f}{\partial \bar{z}_{i_{1}} \cdots \partial \bar{z}_{i_{r}}}\right\|_{L^{p, s}(U)}^{(0)} \quad \text { for } 1 \leq r \leq n, \\
& \|f\|_{L^{p, s}(U)}^{(n)}=\max _{0 \leq r \leq n}\|f\|_{L^{p, s_{(U)}}}^{(0, r)} .
\end{aligned}
$$

If $f=\sum_{\left(\dot{\zeta}_{1}, \ldots, \dot{\zeta}_{q}\right)}^{\prime} f_{\dot{\zeta}_{1} \ldots \dot{\zeta}_{q}} d \bar{z}_{\dot{\zeta}_{1}} \wedge \cdots \wedge d \bar{z}_{\dot{\zeta}_{q}}$ is a $C^{\infty}(0, q)$-form on $U$ where $\sum^{\prime}$, the summation, is over increasing multi-indices, we write $f$ as $\sum_{I}^{\prime} f_{I} d \bar{z}^{I}$ for short $I=$ $\left(i_{1}, \ldots, i_{q}\right)$, and set

$$
\|f\|_{L_{(0, q)}^{p, s}(U)}^{(n)}=\max _{I}\|f\|_{L^{p, s}(U)}^{(n)} .
$$

Then corresponding to [5, Theorem 1], we have the following theorem.

THEOREM 2.1. Let $\Omega$ be a polycylinder in $\mathbb{C}^{n}, 1 \leq p \leq \infty$, and $s$ a nonnegative integer. There is a $k>0$ such that if $f$ is a smooth $\bar{\partial}$-closed $(0, q+1)$-form on $\Omega$ with $\|f\|_{L_{(0, q+1)}^{p, s}(\Omega)}^{(n)}<\infty$, then there is a smooth $(0, q)$-form $u$ on $\Omega$ with $\overline{\partial u}=f$ and

$$
\|u\|_{L_{(0, q)}^{p, s}(\Omega)}^{(n)} \leq K\|f\|_{L_{(0, q+1)}^{p, s}(\Omega)}^{(n)} .
$$

For general locally polycylindrical domains, we combine Theorem 2.1, Leray's isomorphism theorem with bounds, and Dolbeault's isomorphism theorem with bounds to obtain the following result.

THEOREM 2.2. Let $\Omega$ be a locally polycylindrical domain in $\mathbb{C}^{n}, 1 \leq p \leq \infty$, and s a nonnegative integer. If $f$ is a smooth $\bar{\partial}$-closed $(0, q+1)$-form on $\Omega$ with $\|f\|_{L_{(0, q+1)(\Omega)}^{p, s}}^{(n)}<\infty$, then there is a smooth $(0, q)$-form $u$ on $\Omega$ with $\bar{\partial} u=f$ and

$$
\|u\|_{L_{(0, q)(\Omega)}^{p, s}}^{(n)}<\infty .
$$

2.2. We now define the Sobolev-space-holomorphic functions on $\bar{\Omega}$, and state our last theorem in this section which follows from Theorem 2.2.

Let $\Omega$ be a locally polycylindrical domain and $U \neq \varnothing$ a set open in $\bar{\Omega}$, then $B_{\Omega}^{p, s}(U)$ is the Banach space of holomorphic functions $f$ on $\Omega \cap U$ such that $\|f\|_{L^{p, s}(U \cap \Omega)}<\infty$, $1 \leq p \leq \infty$, and $s$ is a nonnegative integer. If $V$ is open in $\bar{\Omega}$ with $\varnothing \neq V \subset U$, the restriction map $\gamma_{V}^{U} . B_{\Omega}^{p, s}(U) \rightarrow B_{\Omega}^{p, s}(V)$ is defined. Then $B_{0}^{p, s}=\left\{B_{\Omega}^{p, s}(U), \gamma_{V}^{U}\right\}$ is the canonical presheaf of $p$-s-Sobolev space holomorphic functions on $\bar{\Omega}$. The associated sheaf $\mathscr{R}_{S}^{p}$ is the sheaf of germs of $p$-s-Sobolev space holomorphic functions on $\bar{\Omega}$.

From Theorem 2.2 we get the following result.

THEOREM 2.3. Let $\Omega$ be a locally polycylindrical domain and $\mathscr{B}_{s}^{p}$ the sheaf of germs of $p$-s-Sobolev space holomorphic functional on $\bar{\Omega}$. Then $H^{q}\left(\bar{\Omega}, \mathscr{P}_{S}^{p}\right)=0$ for $q \geq 1$, $1 \leq p \leq \infty$, and $s$ is a nonnegative integer.

\section{Dolbeault-Grothendieck lemma with bounds}

3.1. In this section, we prove Theorem 2.1. We use the techniques developed in [3]. Since, for the most part we are dealing with smooth objects, the distributional derivatives, used in defining the Sobolev spaces, are ordinary derivatives on the objects. 
However, in the next lemma, which is easy to prove, we need to take distributional derivatives.

LEMMA 3.1. Let $\Omega=D_{1} \times D_{2} \times \cdots \times D_{n}$ be a polycylinder in $\mathbb{C}^{n}$ and let $f \in L^{p, s}(\Omega)$, $1 \leq p \leq \infty$, and $s$ be a nonnegative integer. Define, for $1 \leq j \leq n$,

$$
g_{j}(z)=\int_{D_{j}}\left(\zeta-z_{j}\right)^{-1} f\left(z_{1}, \ldots, z_{j-1}, \zeta, z_{j+1}, \ldots, z_{n}\right) d \zeta \wedge d \bar{\zeta} \quad \text { for a.e. } z \in \Omega .
$$

Then $g_{j} \in L^{p, s}(\Omega)$ and there is a $c>0$ depending on $\Omega, p$, and such that

$$
\left\|g_{j}\right\|_{L^{p, s}(\Omega)} \leq c\|f\|_{L^{p, s}(\Omega)} .
$$

3.2. We can now proceed with the proof of Theorem 2.1. The proof is by induction, the inductive statement being that the theorem is true if $f$ does not involve $d \bar{z}_{k+1}, \ldots, d \bar{z}_{n}$. When $k=0$, there is nothing to prove because then $f$ must be zero.

If $k=n$, then the statement is the theorem. We assume therefore that the theorem is true if $f$ does not involve $d \bar{z}_{k}, d \bar{z}_{k+1}, \ldots, d \bar{z}_{n}$ and assume that

$$
f=d \bar{z}_{k} \wedge g+h
$$

where $g$ is of type $(0, q)$ and $h$ is of type $(0, q+1)$, and $g$ and $h$ are independent of $d \bar{z}_{k}, \ldots, d \bar{z}_{n}$,

$$
g=\sum_{I}^{\prime} g_{I} d \bar{z}^{I}, \quad h=\sum_{J}^{\prime} h_{J} d \bar{z}^{J} .
$$

If $I$ is an increasing multi-index and $j$ is a positive integer not in $I,(I, j)$ is the increasing multi-index obtained by adding $j$ to the integers in $I$, and $\left(I, j_{1}, j_{2}\right)=\left(\left(I, j_{1}\right), j_{2}\right)$, where $j_{1}$ is not in $I$ and $j_{2}$ is not in $\left(I, j_{1}\right)$.

Now on $\Omega$

$$
0=\bar{\partial} f=d \bar{z}_{k} \wedge\left(\sum_{j=1}^{n} d \bar{z}_{j} \wedge\left(\sum_{I}^{\prime} \frac{\partial g_{I}}{\partial \bar{z}_{j}} d \bar{z}^{I}\right)\right)+\sum_{j=1}^{n} d \bar{z}_{j} \wedge\left(\sum_{J}^{\prime} \frac{\partial h_{j}}{\partial \bar{z}_{j}} d \bar{z}^{J}\right),
$$

hence if $I_{0}$ is an increasing multi-index of length $q, 1 \leq j_{0}<k$ and $j_{0}$ is not in $I_{0}$, the coefficient of $d \bar{z}_{k} \wedge d \bar{z}^{\left(I_{0}, j_{0}\right)}$ in $\bar{\partial} f$ is

$$
0=\sum_{\substack{1 \leq j<k \\(I, j)=\left(I_{0}, j_{0}\right)}} \epsilon(I, j) \frac{\partial g_{I}}{\partial \bar{z}_{j}} \pm \frac{\partial h_{J_{0}}}{\partial \bar{z}_{k}}
$$

where $\left(J_{0}, k\right)=\left(I_{0}, j_{0}, k\right), \epsilon(I, j)= \pm 1$, the summation is over $1 \leq j<k$, and $(I, j)=$ $\left(I_{0}, j_{0}\right)$; because

$$
\frac{\partial g_{I}}{\partial \bar{z}_{j}}=0, \quad j>k
$$

this, apart from a factor of \pm 1 , being the coefficient of $d \bar{z}_{k} \wedge d \bar{z}_{j} \wedge d \bar{z}^{I}$ in $\bar{\partial} f=0$. 
In $\Omega$, let

$$
G_{I}(z)=\frac{1}{2 \pi i} \int_{D_{k}}\left(\zeta_{k}-z_{k}\right)^{-1} g_{I}\left(z_{1}, \ldots, z_{k-1}, \zeta_{k}, z_{k+1}, \ldots, z_{n}\right) d \zeta_{k} \wedge d \bar{\zeta}_{k}
$$

Then clearly $G_{I} \in C^{\infty}(\Omega)$ and, by Lemma 3.1, there is a constant $K_{1}>0$ such that

$$
\begin{aligned}
\left\|G_{I}\right\|_{L^{p, s}(\Omega)}^{(n)} & \leq K_{1}\left\|g_{I}\right\|_{L^{p, s}(\Omega)}^{(n)}, \\
\frac{\partial G_{I}}{\partial \bar{z}_{k}}=g_{I}, \quad \frac{\partial G_{I}}{\partial \bar{z}_{j}} & =0, \quad \text { for } j>k .
\end{aligned}
$$

Let $G=\sum_{I}^{\prime} G_{I} d \bar{z}^{I}$, then $\|G\|_{L_{(0, q)(\Omega)}^{p, s}}^{(n)} \leq K_{1}\|f\|_{L_{(0, q+1)}^{p, s}(\Omega)}^{(n)}<\infty$ and

$$
\bar{\partial} G=\sum_{I}^{\prime} \sum_{j=1}^{n} \frac{\partial G_{I}}{\partial \bar{z}_{j}} d \bar{z}_{j} \wedge d \bar{z}^{I}=d \bar{z}_{k} \wedge g+h_{1},
$$

where $h_{1}$ is the sum when $j$ runs from 1 to $k-1$ and it is independent of $d \bar{z}_{k}, \ldots, d \bar{z}_{n}$. Hence $h-h_{1}=f-\bar{\partial} G$ does not involve $d \bar{z}_{k}, \ldots, d \bar{z}_{n}$.

If $I_{0}$ is an increasing multi-index of length $q, 1 \leq j_{0}<k$ and $j_{0}$ is not in $I_{0}$, the coefficient of $d \bar{z}^{\left(I_{0}, j_{0}\right)}$ in $h_{1}$ is

$$
H_{\left(I_{0}, j_{0}\right)}=\sum_{\substack{1 \leq j<k \\(I, j)=\left(I_{0}, j_{0}\right)}} \epsilon(I, j) \frac{\partial G_{I}}{\partial \bar{z}_{j}},
$$

the meaning of the symbols being as in (3.6). From (3.6), it follows that

$$
H_{\left(I_{0}, j_{0}\right)}(z)= \pm \frac{1}{2 \pi i} \int_{D_{k}}\left(\zeta_{k}-z_{k}\right)^{-1} \frac{\partial h_{j_{0}}}{\partial \bar{\zeta}_{k}}\left(z_{1}, \ldots, z_{k-1}, \zeta_{k}, z_{k+1}, \ldots, z_{n}\right) d \zeta_{k} \wedge d \bar{\zeta}_{k}
$$

where $\left(I_{0}, j_{0}, k\right)=\left(J_{0}, k\right)$.

From (3.9), (3.12), and (3.10) and Lemma 3.1, it follows that

$$
\left\|h_{1}\right\|_{L_{(0, q+1)(\Omega)}^{p, s}}^{(n)} \leq K_{2}\|f\|_{L_{(0, q+1)(\Omega)}^{p, s}}^{(n)}
$$

for some constant $K_{2}$, hence

$$
\|f-\bar{\partial} G\|_{L_{(0, q+1)(\Omega)}^{p, s}}^{(n)} \leq\|f\|_{L_{(0, q+1)(\Omega)}^{p, s}}^{(n)}+K_{2}\|f\|_{L_{(0, q+1)(\Omega)}^{p, s}}^{(n)}<\infty .
$$

By the induction hypothesis, since $f-\bar{\partial} G$ does not involve $d \bar{z}_{k}, \ldots, d \bar{z}_{n}$ and $\bar{\partial}(f-$ $\bar{\partial} G)=0$ on $\Omega$, there is a smooth $(0, q)$-form $v$ on $\Omega$, such that $\bar{\partial} v=f-\bar{\partial} G$ on $\Omega$ and

$$
\|v\|_{L_{(0, q)(\Omega)}^{p, s}}^{(n)} \leq K_{3}\|f-\bar{\partial} G\|_{L_{(0, q+1)(\Omega)}^{p, s}} \leq K_{3}\left(1+K_{2}\right)\|f\|_{L_{(0, q+1)(\Omega)}^{p, s}}^{(n)}
$$

for some constant $K_{3}>0$.

Now let $u=v+G$, then $\bar{\partial} u=f$ on $\Omega$ and

$$
\|u\|_{L_{(0, q)(\Omega)}^{p, s}}^{(n)} \leq\left(K_{1}+K_{3}\left(1+K_{2}\right)\right)\|f\|_{L_{(0, q+1)(\Omega)}^{p, s}}^{(n)},
$$

which completes the proof of Theorem 2.1 with $K=\left(K_{1}+K_{3}\left(1+K_{2}\right)\right)$. 


\section{Leray's isomorphism theorem with bounds}

4.1. The formalism for the proof of the theorem which we are going to state in this section, has been used over and over again in [2, 3, 4, 5], we therefore only sketch the proof of that theorem.

Let $O$ be the sheaf of germs of holomorphic functions in $\mathbb{C}^{n}$. If $U \subset \mathbb{C}^{n}$ is open and $\gamma>0$ is an integer, let $\Gamma\left(U, \mathscr{O}^{\gamma}\right)$ be the section of $0^{\gamma}$ on $U$, then

$$
\Gamma_{p, s}\left(U, \mathcal{O}^{\gamma}\right):=\left\{f=\left(f_{1}, \ldots, f_{\gamma}\right) \in \Gamma\left(U, \mathcal{O}^{\gamma}\right):\left\|f_{1}\right\|_{L^{p, s}(U)}+\cdots+\left\|f_{\gamma}\right\|_{L^{p, s}(U)}<\infty\right\} .
$$

If $\mathscr{F}$ is a coherent analytic sheaf on neighborhood of the closure $\bar{U}$ of the polycylinder $U$, then by Cartan's theorem A, there is an exact sequence

$$
\mathcal{O}^{m} \stackrel{\lambda}{\rightarrow} \mathscr{F} \longrightarrow 0
$$

of $\mathcal{O}$-homomorphisms in a neighborhood of $\bar{U}$, where $m$ is a positive integer. The space $L^{p, s}$-bounded sections of $\mathscr{F}_{F}$ over $U, \Gamma_{p, s}(U, \mathscr{F})$ is defined by

$$
\Gamma_{p, s}(U, \mathscr{F}):=\lambda\left(\Gamma_{p, S}\left(U, \mathcal{O}^{m}\right)\right)
$$

It can be shown easily that $\Gamma_{p, s}(U, \mathscr{F})$ does not depend on $\lambda$ and $m$.

Now, let $\Omega$ be a locally polycylindrical domain and let $\mathscr{F}$ be a coherent analytic sheaf in a neighborhood of the closure of $\Omega$. Then $\Omega$ is expressible as the union of a finite number of polycylinders, so let $\mathscr{V}=\left\{U_{j}\right\}_{j \in I}$ be a finite set of polycylinders such that $\Omega=\cup_{j \in I} U_{j}$. We define the $L^{p, s}$-bounded alternate $q$ cochain group $C_{p, s}^{q}(\mathscr{V}, \mathscr{F})$ of the covering $\mathscr{V}$ with values in $\mathscr{F}$ by

$$
C_{p, s}^{q}(\mathscr{V}, \mathscr{F}):=\left\{c=\left(c_{\alpha}\right) \in C^{q}(\mathscr{V}, \mathscr{F}): c_{\alpha} \in \Gamma_{p, s}\left(U_{\alpha}, \mathscr{F}\right), \forall \alpha \in I_{q}^{q+1}\right\},
$$

where $\alpha=\left(\alpha_{0}, \ldots, \alpha_{q}\right), U_{\alpha}=U_{\alpha_{0}} \cap U_{\alpha_{1}} \cap \cdots \cap U_{\alpha_{q}}$, and $C^{q}(\mathscr{V}, \mathscr{F})$ is the alternate $q$ cochain group of the cover $\mathscr{V}$ with values in $\mathscr{F}$.

The coboundary operator

$$
\delta: C^{q}(\mathscr{V}, \mathscr{F}) \longrightarrow C^{q+1}(\mathscr{V}, \mathscr{F})
$$

maps $C_{p, s}^{q}(\mathscr{V}, \mathscr{F})$ into $C_{p, s}^{q+1}(\mathscr{V}, \mathscr{F})$, hence we have the complex

$$
C_{p, s}^{0}(\mathscr{V}, \mathscr{F}) \stackrel{\delta}{\longrightarrow} C_{p, s}^{1}(\mathscr{V}, \mathscr{F}) \stackrel{\delta}{\longrightarrow} \cdots \stackrel{\delta}{\longrightarrow} C_{p, s}^{q}(\mathscr{V}, \mathscr{F}) \stackrel{\delta}{\longrightarrow} C_{p, s}^{q+1}(\mathscr{V}, \mathscr{V}) \stackrel{\delta}{\longrightarrow} \cdots,
$$

and $H_{p, s}^{q}(\mathscr{V}, \mathscr{F})$ is the $q$ th cohomology group of this complex.

We then have the following theorem.

THEOREM 4.1. The natural map

$$
H_{p, s}^{q}(\mathscr{V}, \mathscr{F}) \longrightarrow H^{q}(\Omega, \mathscr{F})
$$

is an isomorphism for $q \geq 0,1 \leq p \leq \infty, s \geq 0$. 
4.2. As in [2, 3, 4, 5], to prove Theorem 4.1, it is enough to show that $H_{p, s}^{q}\left(\mathscr{V}_{|\sigma|}, \mathscr{F}\right)$ $=0$ for all nerves $\sigma$ of the covering $\mathscr{V}$ where $\mathscr{V}_{|\sigma|}=\left\{U_{j} \cap|\sigma|\right\}_{j \in I}$ and $|\sigma|$ is the support of $\sigma$. And since for each nerve $\sigma$ of the covering $\mathscr{V}$ the support of $|\sigma|$ is a polycylinder, we use Theorem 2.1 to show directly that $H_{p, s}^{q}\left(\mathscr{V}_{|\sigma|}, \mathcal{O}\right)=0$ for $q \geq 1$, where as usual, 0 is the sheaf of germs of holomorphic functions in $\mathbb{C}^{n}$. Then from the short exact sequence, $m>1$,

$$
0 \longrightarrow \mathrm{O} \longrightarrow \mathrm{O}^{m} \longrightarrow \mathrm{O}^{m-1} \longrightarrow 0
$$

and induction, we show that $H_{p, s}^{q}\left(\mathscr{V}_{|\sigma|}, \mathcal{O}^{m}\right)=0$ for $q \geq 1,1 \leq p \leq \infty, s \geq 0, m \geq 1$, and $\sigma$ is any nerve of the covering $\mathscr{V}$.

Now, there is a terminating chain of syzygies

$$
0 \longrightarrow \mathrm{O}^{P_{\gamma}} \stackrel{\lambda_{y}}{\longrightarrow} \mathrm{O}^{P_{\gamma-1}} \stackrel{\lambda_{y-1}}{\longrightarrow} \cdots \longrightarrow \mathrm{O}^{P_{0}} \stackrel{\lambda_{0}}{\longrightarrow} \mathscr{F}^{\longrightarrow} 0
$$

in a neighborhood of the closure of $|\sigma|$, where $\gamma$ is a natural number. Using induction on the length $\gamma$ of the terminating chain of syzygies and the fact that $H_{p, s}^{q}\left(\mathscr{V}_{|\sigma|}, \mathcal{O}^{m}\right)=0$ for all $m>0$, we arrive at the desired result that $H_{p, s}^{q}\left(\mathscr{V}_{|\sigma|}, \mathscr{F}\right)=0$, for $q \geq 1,1 \leq p \leq \infty$, $s \geq 0$, and $\sigma$ is any nerve of the covering $\mathscr{V}$, and the proof of the theorem is complete.

\section{Dolbeault's isomorphism theorem with bounds}

5.1. The last thing we need to establish that Theorem 2.2 is Dolbeault's isomorphism theorem with Sobolev bounds. Let $\Omega$ be a locally polycylindrical domain and let $\mathscr{V}=\left\{U_{j}\right\}_{j \in I}$ be a set of polycylinders such that $\Omega=\cup_{j \in I} U_{j}$. Let $\xi^{0, q}$ be the sheaf of germs of $C^{\infty}$ forms of type $(0, q)$ on $\mathbb{C}^{n}$, and $\mathscr{F}^{0, q}$ the sheaf of germs of $\bar{\partial}$-closed $C^{\infty}$ forms of type $(0, q)$ on $\mathbb{C}^{n}$. Define $\hat{\Gamma}_{p, s}\left(\Omega, \xi^{0, q}\right)$ and $\Gamma_{p, s}\left(\Omega, \mathscr{F}^{0, q}\right)$ by

$$
\begin{aligned}
\Gamma_{p, s}\left(\Omega, \xi^{0, q}\right): & =\left\{f \in \Gamma\left(\Omega, \xi^{0, q}\right):\|f\|_{L_{(0, q)}^{p, s}(\Omega)}^{(n)}<\infty\right\}, \\
\Gamma_{p, s}\left(\Omega, \mathscr{F}^{0, q}\right): & =\left\{f \in \Gamma\left(\Omega, \mathscr{F}^{0, q}\right):\|f\|_{L_{(0, q)}^{p, s}(\Omega)}<\infty\right\}, \\
\hat{\Gamma}_{p, s}\left(\Omega, \xi^{0, q}\right): & =\left\{f \in \Gamma_{p, s}\left(\Omega, \xi^{0, q}\right): \bar{\partial} f \in \Gamma_{p, s}\left(\Omega, \mathscr{F}_{0} 0, q+1\right)\right\} .
\end{aligned}
$$

Let $\mathcal{O}$ be the sheaf of germs of holomorphic functions in $\mathbb{C}^{n}$ as before. Then we have the following theorem.

THEOREM 5.1. The group $H_{p, s}^{q}(\mathcal{V}, \mathcal{O})$ is, for $q>0,1 \leq p \leq \infty$, and $s \geq 0$, isomorphic to the quotient space

$$
\frac{\Gamma_{p, s}\left(\Omega, \mathscr{F}^{0, q}\right)}{\bar{\partial} \Gamma_{p, s}\left(\Omega, \xi^{0, q-1}\right) \cap \Gamma_{p, s}\left(\Omega, \xi^{0, q}\right)} .
$$

5.2. To prove Theorem 5.1, with $U_{\alpha}=U_{\alpha_{0}} \cap U_{\alpha_{1}} \cap \cdots \cap U_{\alpha_{q}}$ for a multi-index $\alpha=$ $\left(\alpha_{0}, \ldots, \alpha_{q}\right) \in I^{q+1}$, consider the exact sequence

$$
0 \longrightarrow \Gamma_{p, s}\left(U_{\alpha}, \mathscr{F}^{0, q}\right) \longrightarrow \hat{\Gamma}_{p, s}\left(U_{\alpha}, \xi^{0, q}\right) \stackrel{\bar{\partial}}{\longrightarrow} \Gamma_{p, s}\left(U_{\alpha}, \mathscr{F}^{0, q+1}\right) \longrightarrow 0
$$


The exactness follows from Theorem 2.1. With the cochain groups $C_{p, s}^{\gamma}\left(\mathscr{V}, \mathscr{F}^{0} 0, q\right)$ and $\hat{C}_{p, s}^{\gamma}\left(\mathscr{V}, \xi^{0, q}\right)$ defined from the $\Gamma_{p, s}\left(U_{\alpha}, \mathscr{F}^{0, q}\right)$ and the $\hat{\Gamma}_{p, s}\left(U_{\alpha}, \xi^{0, q}\right)$ in the obvious way, we have the short exact sequence

$$
0 \longrightarrow C_{p, s}^{\gamma}\left(\mathscr{V}, \mathscr{F}^{0, q}\right) \longrightarrow \hat{C}_{p, s}^{\gamma}\left(\mathscr{V}, \xi^{0, q}\right) \stackrel{\grave{\partial}}{\longrightarrow} C_{p, s}^{\gamma}\left(\mathscr{V}, \mathscr{F}^{0, q+1}\right) \longrightarrow 0 .
$$

From which we get the long exact sequence

$$
\begin{aligned}
0 & \longrightarrow \Gamma_{p, s}\left(\Omega, \mathscr{F}^{0, q}\right) \longrightarrow \hat{\Gamma}_{p, s}\left(\Omega, \xi^{0, q}\right) \longrightarrow \Gamma_{p, s}\left(\Omega, \mathscr{F}^{0, q+1}\right) \\
& \longrightarrow H_{p, s}^{1}\left(\mathscr{V}, \mathscr{F}^{0, q}\right) \longrightarrow \hat{H}_{p, s}^{1}\left(\mathscr{V}, \xi^{0, q}\right) \longrightarrow H_{p, s}^{1}\left(\mathscr{V}, \mathscr{F}^{0, q+1}\right) H_{p, s}^{2}\left(\mathscr{V}, \mathscr{F}^{0, q}\right) \longrightarrow \cdots,
\end{aligned}
$$

where $H_{p, s}^{\gamma}\left(\mathscr{V}, \mathscr{F}^{0, q}\right)$ is the $\gamma$ th cohomology group of the complex

$$
C_{p, s}^{0}\left(\mathscr{V}, \mathscr{F}^{0, q}\right) \longrightarrow C_{p, s}^{1}\left(\mathscr{V}, \mathscr{F}^{0, q}\right) \longrightarrow \cdots,
$$

and $\hat{H}_{p, s}^{\gamma}\left(\mathscr{V}, \xi^{0, q}\right)$ is that of the complex

$$
\hat{C}_{p, s}^{0}\left(\mathscr{V}, \xi^{0, q}\right) \longrightarrow \hat{C}_{p, s}^{1}\left(\mathscr{V}, \xi^{0, q}\right) \longrightarrow \cdots
$$

Using the fact that $\xi^{0, q}$ is a fine sheaf, it is easy to show that $\hat{H}_{p, s}^{\gamma}\left(\mathscr{V}, \xi^{0, q}\right)=0$ for $\gamma>0$.

Therefore,

$$
\begin{aligned}
& H_{p, s}^{\gamma}\left(\mathscr{V}, \mathscr{F}^{0, q+1}\right) \approx H_{p, s}^{\gamma+1}\left(\mathscr{V}, \mathscr{F}^{0, q}\right), \quad \gamma \geq 1, \\
& H_{p, s}^{1}\left(\mathscr{V}, \mathscr{F}^{0, q+1}\right) \approx \frac{\Gamma_{p, s}\left(\Omega, \mathscr{F}^{0, q}\right)}{\partial \Gamma_{p, s}\left(\Omega, \xi^{0, q-1}\right) \cap \Gamma_{p, s}\left(\Omega, \xi^{0, q}\right)} .
\end{aligned}
$$

Hence, when $q>0$

$$
H_{p, s}^{q}(\mathscr{V}, \mathcal{O})=H_{p, s}^{q}\left(\mathscr{V}, \mathscr{F}^{0,0}\right) \approx H_{p, s}^{q-1}\left(\mathscr{V}, \mathscr{F}^{0,1}\right) \approx \cdots \approx H_{p, s}^{1}\left(\mathscr{V}, \mathscr{F}^{0, q-1}\right)
$$

This proves Theorem 5.1.

6. Vanishing theorems. We conclude the paper in this section by stating that the same formalism used to establish [5, Theorem 2] works here also in establishing Theorem 2.3, if we have Theorem 2.2 available. Therefore, all we need is to show that, because of Theorem 5.1, we already have Theorem 2.2. This is because the locally polycylindrical domain we are dealing with, is Stein and so by Cartan's theorem, B $H^{q}(\Omega, \mathscr{F})=0$ for all coherent analytic sheaves, for $q \geq 1$. Therefore for $\mathscr{V}$ in Theorem 2.3, $H_{p, s}^{q}(\mathscr{V}, \mathscr{F})=0$ for all coherent analytic sheaves $\mathscr{F}$ defined in a neighborhood of the closure of the locally polycylindrical domain $\Omega$. In particular, $H_{p, s}^{q}(\mathscr{V}, \mathcal{O})=$ 0 for $q \geq 1$ and therefore Theorem 2.3 follows from Theorem 5.1.

REMARK 6.1. Perhaps, we should point out that Theorem 2.3 can be used to solve the Sobolev-Corona problem when the locally polycylindrical domain $\Omega$ has a Lipschitz boundary and $p s>n$, in which case $\Gamma_{p, s}(\Omega, \mathcal{O})$ is an algebra with members extending continuously to the boundary of $\Omega$. 


\section{REFERENCES}

[1] A. Andreotti and W. Stoll, The extension of bounded holomorphic functions from hypersurfaces in a polycylinder, Rice Univ. Studies 56 (1970), no. 2, 199-222.

[2] P. W. Darko, On cohomology with bounds on complex spaces, Atti Accad. Naz. Lincei Rend. Cl. Sci. Fis. Mat. Natur. (8) 60 (1976), no. 3, 189-194.

[3] _ Cohomology with bounds on polycylinders, Rend. Mat. (6) 12 (1979), no. 3-4, 587596.

[4] Cohomology with bounds in $\mathbf{C}^{n}$, Complex Variables Theory Appl. 17 (1992), no. 3-4, 259-263.

[5] P. W. Darko and C. H. Lutterodt, Cohomology with $L^{p}$-bounds on polycylinders, Int. J. Math. Math. Sci. 18 (1995), no. 3, 475-484.

[6] A. Nagel, Cohomology of sheaves of holomorphic functions satisfying boundary conditions on product domains, Trans. Amer. Math. Soc. 172 (1972), 133-141.

Patrick W. Darko: Department of Mathematics and Computer SCience, Lincoln

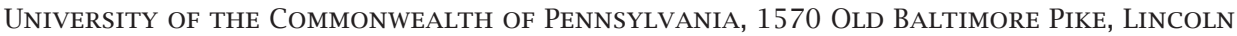
UNIVERSITY, PA 19352, USA

E-mail address: pdarko@1u.1incoln.edu 


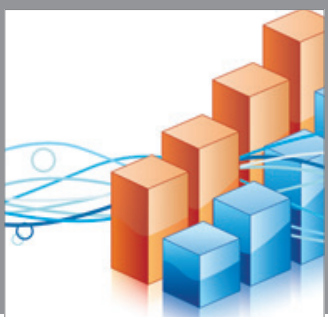

Advances in

Operations Research

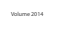

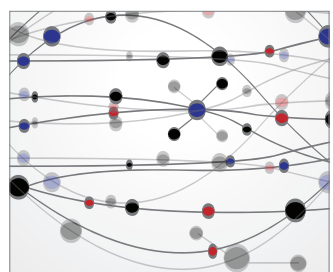

\section{The Scientific} World Journal
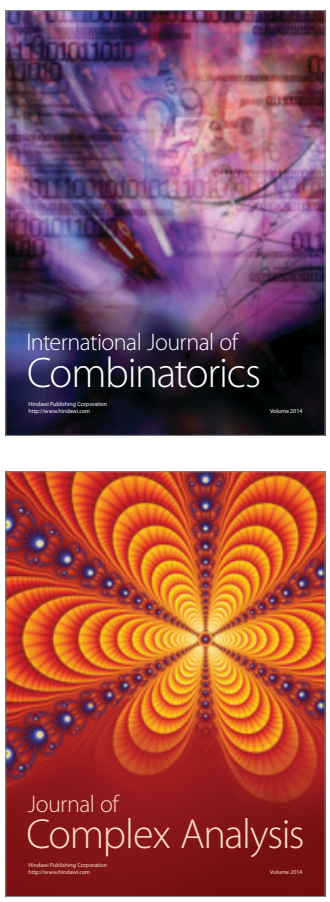

International Journal of

Mathematics and

Mathematical

Sciences
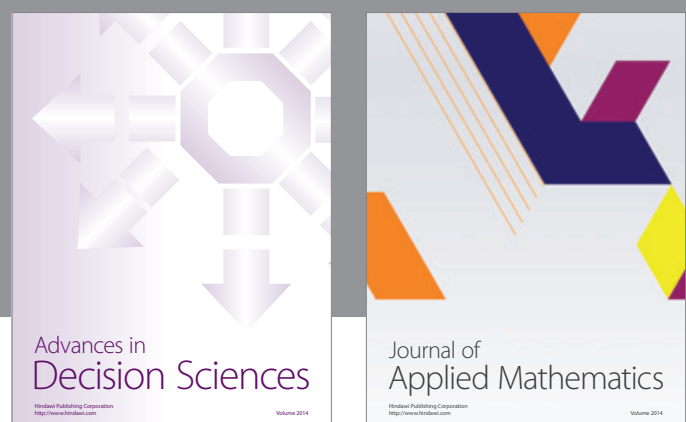

Journal of

Applied Mathematics
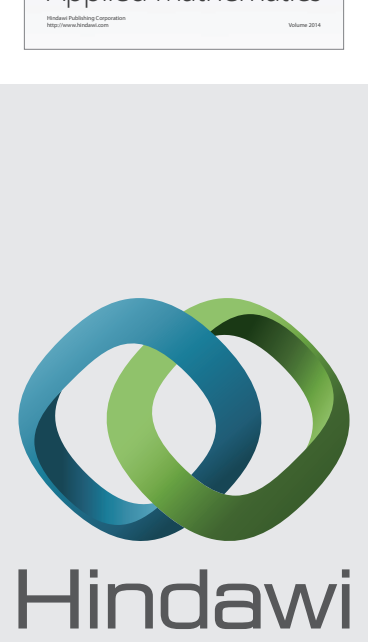

Submit your manuscripts at http://www.hindawi.com
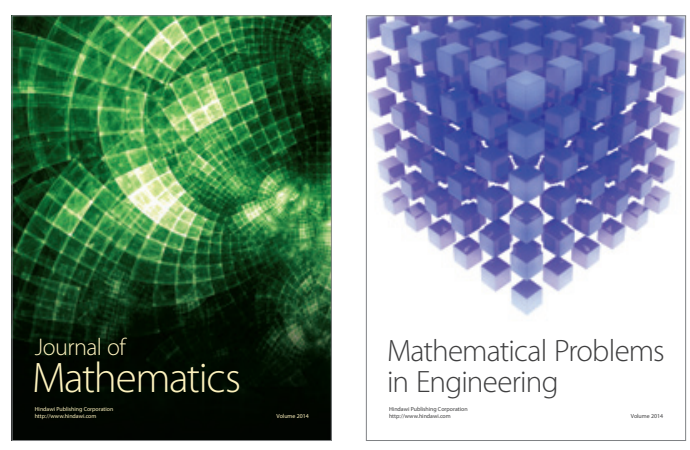

Mathematical Problems in Engineering
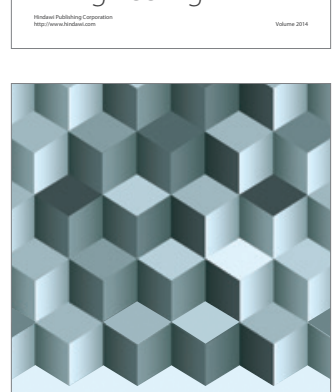

Journal of

Function Spaces
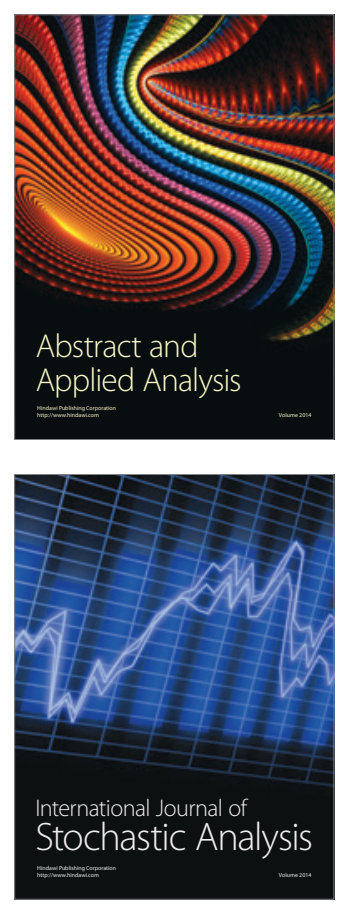

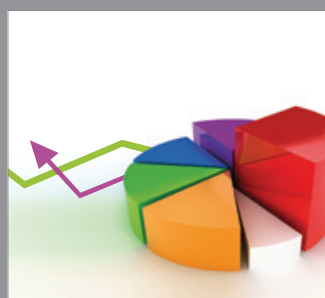

ournal of

Probability and Statistics

Promensencen
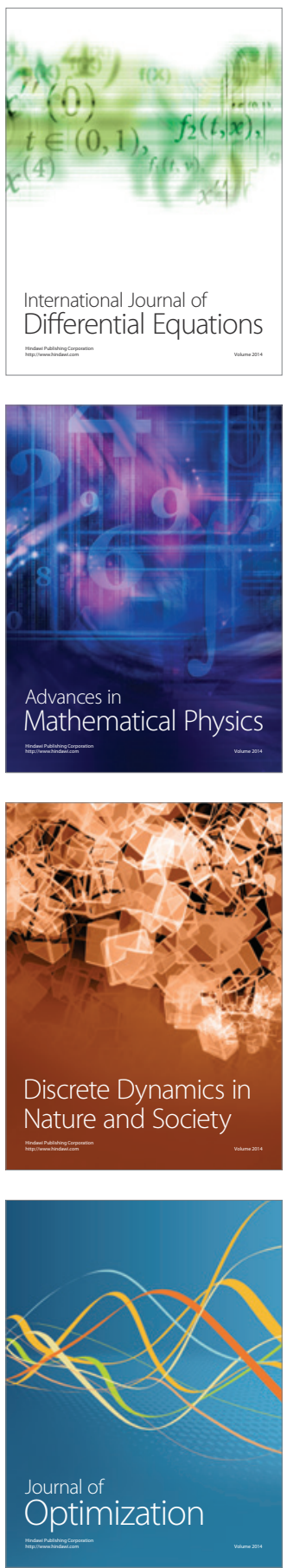